\title{
QUASI-REGULAR SASAKIAN AND K-CONTACT STRUCTURES ON SMALE-BARDEN MANIFOLDS
}

\author{
ALEJANDRO CAÑAS, VICENTE MUÑOZ, MATTHIAS SCHÜTT, AND ALEKSY TRALLE
}

\begin{abstract}
Smale-Barden manifolds are simply-connected closed 5-manifolds. It is an important and difficult question to decide when a Smale-Barden manifold admits a Sasakian or a K-contact structure. The known constructions of Sasakian and K-contact structures are obtained mainly by two techniques. These are either links (Boyer and Galicki), or semi-regular Seifert fibrations over smooth orbifolds (Kollár). Recently, the second named author of this article started the systematic development of quasi-regular Seifert fibrations, that is, over orbifolds which are not necessarily smooth. The present work is devoted to several applications of this theory. First, we develop constructions of a Smale-Barden manifold admitting a quasi-regular Sasakian structure but not a semi-regular K-contact structure. Second, we determine all Smale-Barden manifolds that admit a null Sasakian structure. Finally, we show a counterexample in the realm of cyclic Kähler orbifolds to the algebro-geometric conjecture in [23] that claims that for an algebraic surface with $b_{1}=0$ and $b_{2}>1$ there cannot be $b_{2}$ smooth disjoint complex curves of genus $g>0$ spanning the (rational) homology.
\end{abstract}

\section{INTRODUCTION}

Sasakian and K-contact geometry are topics of great interest for researchers in the fields of differential geometry, algebraic geometry and topology. The main object is defined as follows. Consider a contact co-oriented manifold $(M, \eta)$ with a contact form $\eta$. We say that $(M, \eta)$ admits a Sasakian structure $(M, g, \xi, \eta, J)$ if:

- there exists an endomorphism $J: T M \rightarrow T M$ such that $J^{2}=-\mathrm{Id}+\xi \otimes \eta$, for the Reeb vector field $\xi$ of $\eta$,

- $J$ satisfies the conditions $d \eta(J X, J Y)=d \eta(X, Y)$, for all vector fields $X, Y$ and $d \eta(J X, X)>0$ for all non-zero $X \in \operatorname{ker} \eta$,

- the Reeb vector field $\xi$ is Killing with respect to the Riemannian metric $g(X, Y)=d \eta(J X, Y)+\eta(X) \eta(Y)$,

- the almost complex structure $I$ on the contact cone $C(M)=\left(M \times \mathbb{R}_{+}, t^{2} g+d t^{2}\right)$ defined by $I(X)=J(X), X \in \operatorname{ker} \eta, I(\xi)=t \frac{\partial}{\partial t}, I\left(t \frac{\partial}{\partial t}\right)=-\xi$, is integrable.

If one drops the condition of the integrability of $I$, one obtains a $K$-contact structure.

2010 Mathematics Subject Classification. 53C25, 53D35, 14J28, 14J17.

Key words and phrases. Sasakian, K-contact, Smale-Barden manifold, K3 surface, cyclic orbifold. 
The seminal book [6] shows several important directions of research and still unsolved problems related to manifolds endowed with such structures. One can mention the problems of existence of K-contact/Sasakian structures and the research program of studying topological properties of manifolds endowed with these. Recent papers $[4,8,9,10,11,14,23,25]$ contribute to the topological program. Existence questions were analyzed in the foundational papers of Kollár [18, 19] which showed that studying Sasakian manifolds essentially amounts to constructing Seifert bundles over Kähler or symplectic orbifolds. This technique proved to be very efficient and resulted, for example, in substantial progress in understanding the Smale-Barden manifolds with Sasakian structures [8, 22, 23, 25]. However, several important problems are still not solved, essentially because the known results $[18,19,23,25]$ are obtained for a smaller class of semi-regular Sasakian or K-contact structures, that is, determined by Seifert bundles over smooth orbifolds. These bundles are called semi-regular. If one allows for more general singularities one comes to the notion of a quasi-regular Seifert bundle. The first development of this more general theory was begun by the second author of this article in [22]. In the present paper we further develop the construction techniques for quasi-regular Sasakian or K-contact structures and compare them with the semi-regular case.

A 5-dimensional simply connected manifold $M$ is called a Smale-Barden manifold. These manifolds are classified by their second homology group over $\mathbb{Z}$ and the so-called Barden invariant [1,34]. In more detail, let $M$ be a compact smooth oriented simply connected 5 -manifold. Let us write $H_{2}(M, \mathbb{Z})$ as a direct sum of cyclic group of prime power order

$$
H_{2}(M, \mathbb{Z})=\mathbb{Z}^{k} \oplus\left(\underset{p, i}{\oplus} \mathbb{Z}_{p^{i}}^{c\left(p^{i}\right)}\right)
$$

where $k=b_{2}(M)$. Choose this decomposition in a way that the second StiefelWhitney class map $w_{2}: H_{2}(M, \mathbb{Z}) \rightarrow \mathbb{Z}_{2}$ is zero on all but one summand $\mathbb{Z}_{2^{j}}$. The value of $j$ is unique, it is denoted by $i(M)$ and is called the Barden invariant. The fundamental question arises, which Smale-Barden manifolds admit K-contact or Sasakian structures. In [25] the second and the fourth author of this article obtained classification results for Smale-Barden manifolds with semi-regular Sasakian structures. Thus, one of the aims of this work is to analyze the topic "quasi-regular Sasakian manifolds versus semi-regular". In particular, we ask the following question.

Question 1. Are there Smale-Barden manifolds which carry quasi-regular Sasakian structures but do not carry semi-regular Sasakian structures?

We answer this question in positive in Theorem 26. Moreover, the examples that we provide also satisfy that they do not admit semi-regular K-contact structures. 
In general, we have the following chain of inclusions:

$$
\begin{array}{ccc}
\text { \{Sasakian semi-regular }\} & \subset \quad \text { S Sasakian quasi-regular }\} \\
\cap & \\
\{\text { K-contact semi-regular }\} & \subset\{\text { K-contact quasi-regular }\}
\end{array}
$$

In [8] we have constructed the first Smale-Barden manifold which is K-contact semi-regular but not Sasakian semi-regular. By Corollary 22, we have a Sasakian quasi-regular which is not Sasakian semi-regular. The rightmost inclusion is open (see Question 2 below). In Theorem 26 of this article we show an example of a manifold with a Sasakian quasi-regular structure which does not admit K-contact semi-regular structures, so all other inclusions are strict.

The following problem was posed by Boyer and Galicki [6]:

Question 2. Are there Smale-Barden manifolds with K-contact but no Sasakian structures?

The answer to this question is still not known, although substantial progress has been achieved in [23] and the answer in the class of semi-regular Seifert bundles was found in [8], where it is constructed a Smale-Barden manifold with a semi-regular $\mathrm{K}$-contact structure that does not admit a semi-regular Sasakian structure. The key to the construction of such a semi-regular K-contact manifold $M$ is to find a simply-connected smooth symplectic 4-manifold $X$ with a collection of embedded disjoint symplectic surfaces $D_{i}$ of positive genus $g_{i}>0$ which span the homology $H_{2}(X, \mathbb{Q})$. The manifold $M$ is the Seifert bundle over $X$. Such $M$ does not admit a semi-regular Sasakian structure because such configuration of complex curves is not admissible in a complex surface. Actually, in [23] we find the following conjecture.

Conjecture 3 . There does not exist a smooth complex surface $X$ with $b_{1}=0$ and $b_{2}>1$ such that there are smooth disjoint complex curves $D_{i}$ of positive genus $g_{i}>0$ which span the homology $H_{2}(X, \mathbb{Q})$.

Some cases where the conjecture holds appear in $[8,23]$, which are enough for giving the examples of manifolds not admitting semi-regular Sasakian structures. To find a full answer to Question 2 (that is, in the quasi-regular case) one needs to develop the techniques of constructing symplectic and Kähler non-smooth orbifolds with second homology spanned by symplectic surfaces or complex curves of positive genus. Examples of such orbifolds and the corresponding Seifert bundles are constructed in Section 3. In particular, we show that Conjecture 3 does not hold if we assume $X$ to be a complex cyclic orbifold (Theorem 16).

Our last objective is to settle the problem of existence of null Sasakian structures on Smale-Barden manifolds. Recall that the Reeb vector field $\xi$ on a co-oriented contact manifold $(M, \eta)$ determines a 1-dimensional foliation $\mathcal{F}_{\xi}$ called the characteristic foliation. If we are given a Sasakian manifold $(M, g, \xi, \eta, J)$, then one can define the basic Chern classes $c_{k}\left(\mathcal{F}_{\xi}\right)$ of $\mathcal{F}_{\xi}$ which are elements of the basic cohomology $H_{B}^{2 k}\left(\mathcal{F}_{\xi}\right)$ (see [6, Theorem/Definition 7.5.17]). We say that a Sasakian 
structure is positive (negative) if $c_{1}\left(\mathcal{F}_{\xi}\right)$ can be represented by a positive (negative) definite 1-form. A Sasakian structure is called null, if $c_{1}\left(\mathcal{F}_{\xi}\right)=0$. If none of these cases persists, it is called indefinite.

In [6] it is shown that if a Smale-Barden manifold $M$ admits a null Sasakian structure, then $M$ is homeomorphic to the connected sum of at most 21 copies of $S^{2} \times S^{3}$. Moreover, the authors prove that any $M=\#_{k}\left(S^{2} \times S^{3}\right)$ with $2 \leq k \leq 21$ admits a null Sasakian structure, except, possibly, $b_{2}(M)=2$ and $b_{2}(M)=17$. The following question is left open in [6].

Question 4 ([6, Open Problem 10.3.2]). Find examples of null Sasakian structures on $\#_{2}\left(S^{2} \times S^{3}\right)$ and $\#_{17}\left(S^{2} \times S^{3}\right)$ or show that none can exist.

Note that this question is motivated by applications to $\eta$-Einstein Sasakian geometry [7]. Since the transverse geometry of the characteristic foliation $\mathcal{F}_{\xi}$ is Kähler, one can understand Sasakian geometry in analogy with Kähler geometry. In particular, a null Sasakian structure can be understood as a transversal Calabi-Yau structure. Therefore, manifolds with null Sasakian structures are odd-dimensional analogues of the Calabi-Yau spaces. Also, every null Sasakian structure can be deformed to an $\eta$-Einstein structure (see [7] for a discussion of possible applications in physics).

The case $\#_{17}\left(S^{2} \times S^{3}\right)$ was settled in [12] using a generalization of the initial approach of Boyer and Galicki via Seifert bundles over weighted surface complete intersections in weighted projective spaces $[16,28]$. Our approach is different and enables us to provide a complete solution of the problem. This solution illustrates our general techniques. We answer Question 4 in the positive:

Theorem 5. Any $M=\#_{k}\left(S^{2} \times S^{3}\right), 2 \leq k \leq 21$ admits a null Sasakian structure.

Note that the formulation of Theorem A in the introduction of [12] is incorrect, the correct formulation should be " $\#{ }_{k}\left(S^{2} \times S^{3}\right)$ with $3 \leq k \leq 21$ admits a null Sasakian structure".

Our basic reference containing elliptic surfaces, complex surfaces, and desingularization process is [13].

Acknowledgements. The first author is supported by a $\mathrm{PhD}$ grant from Universidad de Málaga. The second author was partially supported by Project MINECO (Spain) PGC2018-095448-B-I00. The fourth author was supported by the National Science Center (Poland), grant NCN no. 2018/31/D/ST1/00053. The fourth author started working on the topic during his stay at the Institut de Hautes Études Scientifiques at Bur-sur-Yvette. He is grateful to the Institute for the support and the stimulating research atmosphere. 


\section{Preliminaries on Seifert Bundles}

We freely use the notion of cyclic orbifolds [6, 18, 19, 22]. Our basic reference will be $[22]$.

Definition 6 ([22]). A cyclic singular symplectic (Kähler) manifold is a symplectic cyclic 4-orbifold whose isotropy set is of dimension zero (that is, a finite set $P$ of points, called the singular set).

For a cyclic singular symplectic 4-manifold a singular point is an isolated isotropy point $x \in P \subset X$. A local model around $x$ is of the form $\mathbb{C}^{2} / \mathbb{Z}_{d}$, where $\xi=$ $\exp (2 \pi i / d)$ acts as $\xi \cdot\left(z_{1}, z_{2}\right)=\left(\xi^{e_{1}} z_{1}, \xi^{e_{2}} z_{2}\right)$, where $\operatorname{gcd}\left(e_{1}, d\right)=\operatorname{gcd}\left(e_{2}, d\right)=1$. We will write $d=d(x)$.

Definition 7 ([22]). A sing-symplectic surface is a symplectic 2-orbifold $D \subset X$ such that if $x \in D$ is a singular point, then $D$ is fixed by the isotropy subgroup. Two sing-symplectic surfaces $D_{1}, D_{2}$ intersect nicely if at every intersection point $x \in D_{1} \cap D_{2}$ there is an adapted Darboux chart $\left(z_{1}, z_{2}\right)$ centered at $x$ such that $D_{1}=\left\{\left(z_{1}, 0\right)\right\}$ and $D_{2}=\left\{\left(0, z_{2}\right)\right\}$ in a model $\mathbb{C}^{2} / \mathbb{Z}_{d}$, where $\mathbb{Z}_{d}<U(1) \times U(1)$.

One can construct cyclic symplectic or Kähler orbifolds using the result below.

Proposition 8 ([22]). Let $X$ be a cyclic singular 4-manifold with the set of singular points $P$. Let $D_{i}$ be embedded sing-symplectic surfaces intersecting nicely, and take coefficients $m_{i}>1$ such that $\operatorname{gcd}\left(m_{i}, m_{j}\right)=1$, if $D_{i}$ and $D_{j}$ have a nonempty intersection. Then there exists an orbifold $X$ with isotropy surfaces $D_{i}$ of multiplicities $m_{i}$, and singular points $x \in P$ of multiplicity $m=d(x) \prod_{i \in I_{x}} m_{i}$, $I_{x}=\left\{i \mid x \in D_{i}\right\}$.

Our basic tool is a Seifert bundle over a sing-symplectic orbifold.

Definition 9. Let $X$ be a cyclic, oriented $n$-orbifold. A Seifert bundle over $X$ is an oriented $(n+1)$-dimensional manifold $M$ equipped with a smooth $S^{1}$-action and a continuous map $\pi: M \rightarrow X$ such that for an orbifold chart $\left(U, \tilde{U}, \mathbb{Z}_{m}, \varphi\right)$, there is a commutative diagram

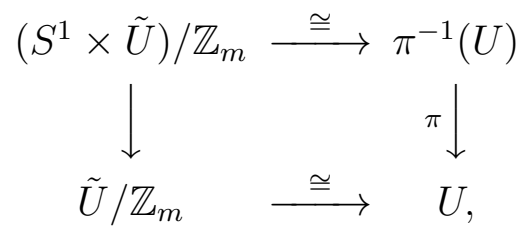

where the action of $\mathbb{Z}_{m}$ on $S^{1}$ is by multiplication by $\xi=\exp (2 \pi i / m)$ and the top diffeomorphism is $S^{1}$-equivariant.

The basic invariant of the Seifert bundle is the orbifold first Chern class.

Definition 10. For a Seifert bundle $\pi: M \rightarrow X$, we define its first Chern class as follows. Let $l=\operatorname{lcm}(m(x) \mid x \in X)$. Denote by $M / l$ the quotient of $M$ by 
$\mathbb{Z}_{l} \subset S^{1}$. Then $M / l \rightarrow X$ is a circle fiber bundle with the first Chern class $c_{1}(M / l) \in H^{2}(X, \mathbb{Z})$. Define

$$
c_{1}(M)=\frac{1}{l} c_{1}(M / l) \in H^{2}(X, \mathbb{Q}) .
$$

Definition 11. We say that an element $a$ in a free abelian group $A$ is primitive, if it cannot be represented as $a=k b$ with a non-trivial $b \in A, k \in \mathbb{N}$.

Here is the main construction tool of this paper.

Theorem 12 ([22, Lemma 39]). Let $(X, \omega)$ be a cyclic symplectic 4-orbifold with a collection of embedded symplectic surfaces $D_{i}$ intersecting nicely, and integer numbers $m_{i}>1$, with $\operatorname{gcd}\left(m_{i}, m_{j}\right)=1$ whenever $D_{i} \cap D_{j} \neq \emptyset$. Assume that there are local invariants $\left(m_{i}, j_{i}, j_{x}\right), x \in P$. Let $b_{i}$ be integers such that $j_{i} b_{i} \equiv$ $1\left(\bmod m_{i}\right), m=\operatorname{lcm}\left(m_{i}\right)$. Then there is a Seifert bundle $\pi: M \rightarrow X$ such that

(1) It has the first orbifold Chern class $c_{1}(M)=[\hat{\omega}]$ for an orbifold symplectic form $\hat{\omega}$.

(2) If $\sum_{i} \frac{b_{i} m}{m_{i}}\left[D_{i}\right] \in H^{2}(X-P, \mathbb{Z})$ is primitive and the second Betti number $b_{2}(X) \geq 3$, then we can further have that $c_{1}(M / m) \in H^{2}(X-P, \mathbb{Z})$ is primitive.

This combines with the folllowing basic result for characterizing K-contact and Sasakian structures.

Theorem 13 ([6, Theorems 7.5.1, 7.5.2]). Let $(M, g, \xi, \eta, J)$ be a quasi-regular Sasakian manifold. Then the space of leaves $X$ of the foliation determined by the Reeb field $\xi$ has a natural structure of a cyclic Khler orbifold, and the projection $M \rightarrow X$ is a Seifert bundle. Conversely, if $(X, \omega)$ is a Khler cyclic orbifold and $M$ is the total space of the Seifert bundle determined by the class [ $\omega]$, then $M$ admits a quasi-regular Sasakian structure.

In the same way, one can characterize quasi-regular K-contact manifolds considering symplectic orbifolds instead of Kähler ones [23, Theorems 19 and 21].

Note that by [30], any manifold which admits a Sasakian or K-contact structure, admits a quasi-regular one. Therefore, since we are interested in the existence questions, we can and we will assume that we are dealing with quasi-regular structures.

Finally, let us formulate a result which guarantees that the Seifert bundle $M \rightarrow$ $X$ constructed in Theorem 12 has $H_{1}(M, \mathbb{Z})=0$.

Theorem 14 ([22, Theorem 36]). Suppose that $\pi: M \rightarrow X$ is a quasi-regular Seifert bundle over a cyclic orbifold $X$ with isotropy surfaces $D_{i}$ and set of singular points $P$. Let $m=\operatorname{lcm}\left(m_{i}\right)$. Then $H_{1}(M, \mathbb{Z})=0$ if and only if

(1) $H_{1}(X, \mathbb{Z})=0$, 
(2) $H^{2}(X, \mathbb{Z}) \rightarrow \oplus H^{2}\left(D_{i}, \mathbb{Z}_{m_{i}}\right)$ is surjective,

(3) $c_{1}(M / m) \in H^{2}(X-P, \mathbb{Z})$ is a primitive class.

Moreover, $H_{2}(M, \mathbb{Z})=\mathbb{Z}^{k} \oplus\left(\underset{i}{\oplus} \mathbb{Z}_{m_{i}}^{2 g_{i}}\right), g_{i}=$ genus of $D_{i}, k+1=b_{2}(X)$.

For the convenience of references we will also interchangeably follow notation and terminology of $[6]$ and $[18,19]$. If $X$ is a cyclic orbifold with singular set $P$ and a family of surfaces $D_{i}$ we will say that we are given a divisor $\cup_{i} D_{i}$, with multiplicities $m_{i}>1$. The formal sum $\Delta=\sum_{i}\left(1-\frac{1}{m_{i}}\right) D_{i}$ will be called the branch divisor. By definition, the orbifold fundamental group $\pi_{1}^{\text {orb }}(X)$ is defined as

$$
\pi_{1}^{\text {orb }}(X)=\pi_{1}(X-(\Delta \cup P)) /\left\langle\gamma_{i}^{m_{i}}=1\right\rangle,
$$

where $\left\langle\gamma_{i}^{m_{i}}=1\right\rangle$ denotes the following relation on $\pi_{1}(X-(P \cup \Delta))$ : for any small loop $\gamma_{i}$ around a surface $D_{i}$ in the branch divisor, one has $\gamma_{i}^{m_{i}}=1$. We will systematically use without notice the following exact sequence (see the general formulation in [6, Theorem 4.3.18]) . If $M \rightarrow X$ is a Seifert fibration, then there is an exact sequence

$$
\cdots \rightarrow \pi_{1}\left(S^{1}\right)=\mathbb{Z} \rightarrow \pi_{1}(M) \rightarrow \pi_{1}^{\text {orb }}(X) \rightarrow 1 .
$$

It follows that if $H_{1}(M, \mathbb{Z})=0$ and $\pi_{1}^{\text {orb }}(X)=1$, then $M$ must be simply connected. We will use this observation without further notice.

\section{EXAMPLES OF KÄHLER CYCLIC ORBIFOLDS}

The following appears in [2, Section III.5].

Lemma 15. Consider the action of the cyclic group $\mathbb{Z}_{m}$ on $\mathbb{C}^{2}$ given by $\left(z_{1}, z_{2}\right) \mapsto$ $\left(\xi z_{1}, \xi^{r} z_{2}\right)$, where $\xi=e^{2 \pi i / m}, 0<r<m$ and $\operatorname{gcd}(r, m)=1$. Then write $a$ continuous fraction

$$
\frac{m}{r}=\left[b_{1}, \ldots, b_{l}\right]=b_{1}-\frac{1}{b_{2}-\frac{1}{b_{3}-\ldots}}
$$

The resolution of $\mathbb{C}^{2} / \mathbb{Z}_{m}$ has an exceptional divisor formed by a chain of smooth rational curves of self-intersections $-b_{1},-b_{2}, \ldots,-b_{l}$.

Conversely, let $X$ be a smooth surface containing a chain of smooth rational curves $E_{1}, \ldots, E_{l}$ of self-intersections $-b_{1},-b_{2}, \ldots,-b_{l}$, with all $b_{i} \geq 2$, intersecting transversally (so that $E_{i} \cap E_{i+1}$ are nodes, $i=1, \ldots, l-1$ ). Let $\pi: X \rightarrow \bar{X}$ be the contraction of $E=E_{1} \cup \ldots \cup E_{l}$. Then $\bar{X}$ has a cyclic singularity at $p=\pi(E)$, with an action given by Lemma 15. Moreover, if $D$ is a curve intersecting tranversally a tail of the chain (that is, either $E_{1}$ or $E_{l}$ at a non-nodal point), then the push down curve $\bar{D}=\pi(D)$ is a sing-complex curve (as in Definition 7). This can be seen as follows. By [29, Corollary 2.5], if $z_{1}, z_{2}$ are the coordinates of $\mathbb{C}^{2}$, then the quotient $Z=\mathbb{C}^{2} / \mathbb{Z}_{m}$ is described by coordinates $u_{0}, u_{1}, \ldots, u_{l+1}$, where

$$
u_{0}=z_{1}^{m}, \quad u_{i-1} u_{i+1}=u_{i}^{a_{i}} \text { for } i=1, \ldots, l, \quad u_{l+1}=z_{2}^{m},
$$


where $\frac{m}{r-m}=\left[a_{1}, \ldots, a_{l}\right]$. The resolution is obtained by a (weighted) blow-up at the origin $\left(u_{0}, u_{1}, \ldots, u_{l+1}\right)=(0, \ldots, 0)$ of $Z \subset \mathbb{C}^{l+2}$. The exceptional divisor is the subset $E$ of a suitable weighted projective space $\mathbb{P}_{w}^{l+1}$, with coordinates $\left[w_{0}, w_{1}, \ldots, w_{l+1}\right]$ parametrized by the equations $w_{i-1} w_{i+1}=w_{i}^{a_{i}}, i=1, \ldots, l$. The divisor $E_{i}$ is given by the equations $w_{0}=\ldots=w_{i-2}=0, w_{i+2}=\ldots=w_{l+1}=0$. The strict transform of $\left\{z_{1}=0\right\}$ only intersects $E_{1}$, and the strict transform of $\left\{z_{2}=0\right\}$ only intersects $E_{l}$.

Our aim now is to construct a Kähler cyclic orbifold with the second homology generated by elliptic curves. This will provide a counterexample to Conjecture 3 in the case where $X$ is assumed to be a complex cyclic orbifold.

Theorem 16. For any $b \geq 1$, there is a Kähler cyclic orbifold $X$ with $b_{1}=0$, $b_{2}=b$ and $b$ disjoint curves, all of genus 1 , whose classes span $H^{2}(X, \mathbb{Q})$.

Proof. Consider a regular cubic $C$ inside the projective plane $\mathbb{C} P^{2}$. In particular, it has genus 1. Consider now the following short exact sequence of sheaves,

$$
0 \rightarrow \mathcal{O}_{\mathbb{C} P^{2}} \rightarrow \mathcal{O}_{\mathbb{C} P^{2}}(C) \rightarrow \mathcal{O}_{C}(C) \rightarrow 0 .
$$

It is known that $h^{0}\left(\mathcal{O}_{\mathbb{C} P^{2}}\right)=1, h^{1}\left(\mathcal{O}_{\mathbb{C} P^{2}}\right)=0$ and $h^{0}\left(\mathcal{O}_{\mathbb{C} P^{2}}(C)\right)=h^{0}\left(\mathcal{O}_{\mathbb{C} P^{2}}(3)\right)=$ 10. Therefore, we get the following exact short sequence in cohomology,

$$
0 \rightarrow H^{0}\left(\mathcal{O}_{\mathbb{C} P^{2}}\right) \rightarrow H^{0}\left(\mathcal{O}_{\mathbb{C} P^{2}}(C)\right) \rightarrow H^{0}\left(\mathcal{O}_{C}(C)\right) \rightarrow 0
$$

from where we deduce that $h^{0}\left(\mathcal{O}_{C}(C)\right)=9$. This implies that the linear system $\left|\mathcal{O}_{C}(C)\right|$ has dimension 8 . Hence, for any 8 points chosen in $C$, we can find a cubic $C^{\prime} \in|C|$ which intersects $C$ at those points.

Take $p \in C$ and $C^{\prime}$ a cubic such that $C \cdot C^{\prime}=8 p+q$ for some $q \in C$. This defines a map $\varphi: C \rightarrow C$ given by $q=\varphi(p)$. We look for a fixed point of $\varphi$. We describe the map in an alternative way. Fix some base-point $p_{0} \in C$. This produces an isomorphism

$$
F: C \rightarrow \operatorname{Jac} C, \quad F(p)=p-p_{0}
$$

where Jac $C$ is the Jacobian of degree 0 divisors. Consider the divisor $D_{0}=8 p_{0}+$ $\varphi\left(p_{0}\right) \in\left|\mathcal{O}_{C}(C)\right|$. All divisors $8 p+\varphi(p)$ are equivalent, hence $\varphi(p) \equiv D_{0}-8 p$, so

$$
\begin{aligned}
F(\varphi(p)) & =\varphi(p)-p_{0}=D_{0}-8 p-p_{0}= \\
& =\varphi\left(p_{0}\right)-p_{0}-8\left(p-p_{0}\right)=F\left(\varphi\left(p_{0}\right)\right)-8 F(p) .
\end{aligned}
$$

Hence

$$
\varphi(p)=F^{-1}\left(F\left(\varphi\left(p_{0}\right)\right)-8 F(p)\right) .
$$

As multiplication (by $k=-8$ ) and addition in $\operatorname{Jac} C$ are morphisms, then $\varphi$ is a morphism.

To find a fixed point $p \in C$, we note that $\varphi(p)=p$ is equivalent to $F(p)=$ $F(\varphi(p))=F\left(\varphi\left(p_{0}\right)\right)-8 F(p)$, i.e. $9 F(p)=F\left(\varphi\left(p_{0}\right)\right)$. The map

$$
m_{9}: \operatorname{Jac} C \rightarrow \operatorname{Jac} C, \quad m_{9}(D)=9 D
$$


given by multiplication by 9 in the divisors of degree 0 , is a map of degree $81=9^{2}$. The divisor $s_{0}=F\left(\varphi\left(p_{0}\right)\right)$ has 81 preimages $r_{i} \in \operatorname{Jac} C, 1 \leq i \leq 81$. Then $p_{i}=F^{-1}\left(r_{i}\right)$ are the 81 solutions to the equation $9 F\left(p_{i}\right)=9 r_{i}=s_{0}=F\left(\varphi\left(p_{0}\right)\right)$, that is, to the equation $\varphi\left(p_{i}\right)=p_{i}$.

Now fix one of these 81 points $p=p_{i}$. Then take a cubic $C^{\prime}$ such that $C \cdot C^{\prime}=9 p$. Take sections $s, s^{\prime} \in H^{0}\left(\mathcal{O}_{\mathbb{C} P^{2}}(3)\right)$ with $C$ and $C^{\prime}$ as zero loci. We then get a pencil $\left|\left\langle s, s^{\prime}\right\rangle\right| \subset\left|\mathcal{O}_{\mathbb{C} P^{2}}(3)\right|$ of cubics intersecting at $p$ with multiplicity 9. Choose $b$ regular cubics inside this pencil, $C_{1}, \ldots, C_{b}$. These all have genus 1 , self-intersection $C_{i}^{2}=9$ and any two of them intersect only at $p$, with multiplicity 9 .

Blow-up the plane $\mathbb{C} P^{2}, 9$ times at $p$ and denote the resulting surface by $\widetilde{\mathbb{C} P^{2}}$. That is, we blow-up at $p$, then at the intersection point of the strict transforms of the $C_{i}$, and so on. We obtain a chain of 9 rational curves $E_{1}, \ldots, E_{9}$. All the exceptional divisors $E_{1}, \ldots, E_{8}$ have self-intersection $E_{j}^{2}=-2, j=1, \ldots, 8$. The last exceptional divisor $E_{9}$ satisfies $E_{9}^{2}=-1$, and the strict transforms of the curves $C_{1}, \ldots, C_{b}$, that we denote $\tilde{C}_{1}, \ldots, \tilde{C}_{b}$, are now pairwise disjoint, each of them intersects $E_{9}$ at one point $x_{i}$, and all are disjoint from $E_{1}, \ldots, E_{8}$. Note that $\tilde{C}_{i}^{2}=0,1 \leq i \leq b$. Blow-up $\widetilde{\mathbb{C} P^{2}}$ at the points $x_{2}, \ldots, x_{b}$ and denote by $\tilde{X}$ this new surface. Since we obtained $\tilde{X}$ from $\mathbb{C} P^{2}$ by $9+(b-1)$ blow-ups, $b_{1}(\tilde{X})=0$ and $b_{2}(\tilde{X})=9+b$. Denote by $\tilde{E}_{9}$ the strict transform of $E_{9}$ and by $D_{i}$ the strict transform of $\tilde{C}_{i}, 2 \leq i \leq b$. Now $\tilde{E}_{9}^{2}=-b, D_{i}^{2}=-1,2 \leq i \leq b, \tilde{C}_{1}^{2}=0$. It is clear that $\tilde{C}_{1}, D_{2}, \ldots, D_{b}, E_{1}, \ldots, E_{8}, \tilde{E}_{9}$ form a basis of $H^{2}(X, \mathbb{Q})$.

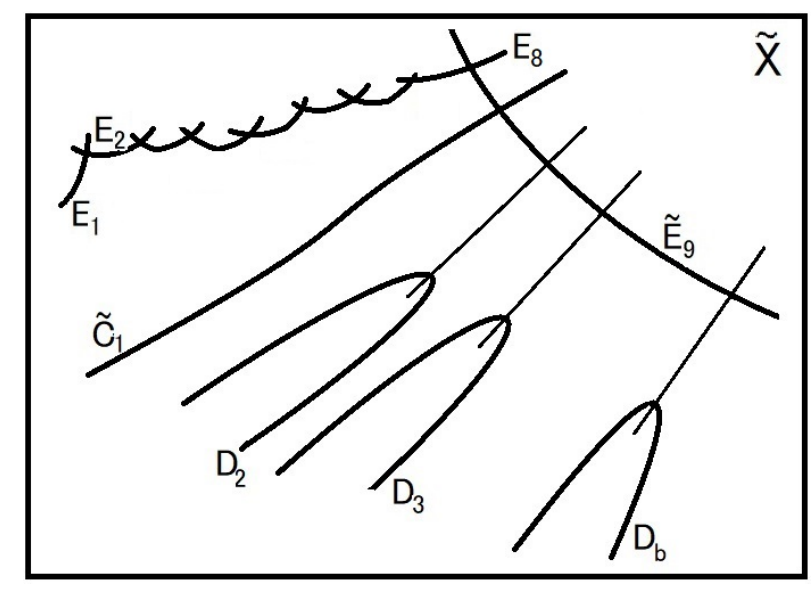

Now we contract the chain of $(-2)$-curves given by $E_{1}, \ldots, E_{8}$, extended by the $(-b)$-curve $\tilde{E}_{9}$. By Lemma 15 , this produces a cyclic singularity. As $\left[b, 2,{ }^{(8)} ., 2\right]=$ $\frac{9 b-8}{9}$, the singularity is modelled on $\mathbb{C}^{2} / \mathbb{Z}_{9 b-8}$ with the action of $\xi=\exp (2 \pi i /(9 b-$ $8)$ ) via $\left(z_{1}, z_{2}\right) \mapsto\left(\xi z_{1}, \xi^{9} z_{2}\right)$. The result is a cyclic orbifold $X$ with $b_{1}(X)=0$, $b_{2}(X)=b$ and $b$ disjoint curves $D_{1}, D_{2}, \ldots D_{b}$ all of genus 1 . Here $D_{1}$ is the push down of $\tilde{C}_{1}$ and has self-intersection $D_{1}^{2}=\tilde{C}_{1}^{2}+\frac{1}{9 b-8}=\frac{1}{9 b-8}>0$. 
Remark 17. We can simplify the construction in the proof of Theorem 16. Take a cubic $C$ and a point $p \in C$ which is an inflection. Then let $L$ be the tangent line, and $C^{\prime}=3 L$ is a cubic and satisfies that $C \cap 3 L=9 p$. Now take the pencil generated by them $|\langle C, 3 L\rangle|$. The generic curve is smooth because there is a curve, namely $C$, that is smooth. All of them intersect at $p$ with multiplicity 9 .

The construction in Theorem 16 produces 81 points. As the number of inflections is 9 , there are pencils as in Theorem 16 which do not come from inflections.

Remark 18. The surface $\widetilde{\mathbb{C} P^{2}}$ above is a so-called extremal rational elliptic surface, i.e. with finite group of sections. Similar constructions can be carried out for any other extremal rational elliptic surface (as classified in [21]). Then one can always contract suitable configurations of eight $(-2)$-curves inside the singular fibres (like $\left.E_{1}, \ldots, E_{8}\right)$. The role of $E_{9}$ is played by some section.

Remark 19. On Enriques surfaces, the same arrangement of the singular fibres can be achieved as on the rational elliptic surfaces in Remark 18 . The section $E_{9}$, however, has to be replaced by a rational curve with $b-1$ nodes, serving as a bisection of the elliptic fibration, which becomes smooth exactly after blowing up the intersection points with $b-1$ regular fibres $C_{2}, \ldots, C_{b}$. Such surfaces can be constructed systematically using the base change approach from [15]. Unlike $X$ below in Proposition 20, they will not have trivial $\pi_{1}^{\text {orb }}$.

We put an orbifold structure on the surface $X$ constructed in Theorem 16 by assigning coefficients $m_{i}$ to each $D_{i}, i=1, \ldots, b$, using Proposition 8. Let us compute the orbifold fundamental group.

Proposition 20. We have $\pi_{1}^{\text {orb }}(X)=1$.

Proof. Let $\pi: \tilde{X} \rightarrow \mathbb{C} P^{2}$ be the result of the successive blow-ups of $\mathbb{C} P^{2}$, with the tori $\tilde{C}_{1}, D_{2}, \ldots, D_{b}$ and exceptional curves $E_{1}, \ldots, E_{8}, \tilde{E}_{9}$ and $E_{10}, \ldots, E_{8+b}$ coming from the blow-ups at $x_{2}, \ldots, x_{b}$. We denote $E=E_{1} \cup \ldots \cup E_{8} \cup \tilde{E}_{9} \cup E_{10} \cup \ldots \cup$ $E_{8+b}$. The curves $C_{1}=\pi\left(\tilde{C}_{1}\right), C_{i}=\pi\left(D_{i}\right), i=2, \ldots, b$, are smooth cubic curves intersecting at the point $p=\pi(E)$. Denote $D^{\prime}=\tilde{C}_{1} \cup D_{2} \cup \ldots \cup D_{b} \cup E \subset \tilde{X}$ and $C=\cup C_{i} \subset \mathbb{C} P^{2}$. Then there is an isomorphism

$$
\tilde{X}-U\left(D^{\prime}\right) \cong \mathbb{C} P^{2}-U(C),
$$

where $U\left(D^{\prime}\right), U(C)$ denote small tubular neighbourhoods of $D^{\prime}, C$, respectively. The fundamental group of $C_{i}$ is generated by two loops $\alpha_{i}, \beta_{i}$. The loops $\alpha_{i}, \beta_{i}$ are homotopic to $\alpha_{j}, \beta_{j}$ in $U(C)$ (it is enough to construct $C_{i}$ close together and generic in the pencil). Moreover, they are contractible in $U(C)$. For this it is enough to take a curve $C_{1}$ close to a cuspidal curve, the point $p$ well away from the cusp, and all other curves $C_{i}$ close to $C_{1}$.

Now consider the blow-down map

$$
\varpi: \tilde{X} \rightarrow X
$$


and the images $D_{1}=\varpi\left(\tilde{C}_{1}\right), D_{i}=\varpi\left(D_{i}\right), i=2, \ldots, b$. Recall that $D_{1}$ contains the singular point $q=\varpi(F)$, where $F=E_{1} \cup \ldots \cup E_{8} \cup \tilde{E}_{9}$. Let $2 \leq i \leq b$. The boundary $\partial U\left(D_{i}\right)$ is generated by $\alpha_{i}, \beta_{i}$ and a loop $\gamma_{i}$ around $D_{i}$. As $D_{i}^{2}=-1$, we have $\gamma_{i}^{-1}=\left[\alpha_{i}, \beta_{i}\right]$. As $\alpha_{i}, \beta_{i}$ is contractible in $\tilde{X}-U(D)$, hence in $X-U\left(\cup D_{i}\right)=$ $\tilde{X}-U(D)$, where $D=\tilde{C}_{1} \cup D_{2} \cup \ldots \cup D_{b} \cup F \subset D^{\prime}$, since $F \subset E$. There is a surjective map

$$
\pi_{1}\left(\tilde{X}-U\left(D^{\prime}\right)\right)=\pi_{1}\left(\mathbb{C} P^{2}-U(C)\right) \longrightarrow \pi_{1}(\tilde{X}-U(D))=\pi_{1}\left(X-U\left(\cup D_{i}\right)\right) .
$$

Therefore the group $\pi_{1}\left(X-U\left(\cup D_{i}\right)\right)$ is generated by a loop around $D_{1}$, and by a loop going around a neighbourhood of $q$, which is the image of a loop in $\partial U(F)$. The point $q$ is modeled on a space $\mathbb{C}^{2} / \mathbb{Z}_{d}$ and the link is a lens space. Let $x \in \pi_{1}\left(\mathbb{C}^{2} / \mathbb{Z}_{d}-\{q\}\right) \cong \mathbb{Z}_{d}$ be a generator, where $d=9 b-8>1$ is the order of the singular point. The orbifold fundamental group of $D_{1}$ is

$$
\pi_{1}^{\text {orb }}\left(D_{1}\right)=\left\langle\alpha_{1}, \beta_{1}, x \mid\left[\alpha_{1}, \beta_{1}\right] x=1, x^{d}=1\right\rangle .
$$

There is a Seifert fibration $S^{1} \rightarrow \partial U\left(D_{1}\right) \rightarrow D_{1}$, which gives an exact sequence

$$
0 \rightarrow \mathbb{Z}\left\langle\gamma_{1}\right\rangle \rightarrow \pi_{1}\left(\partial U\left(D_{1}\right)\right) \rightarrow \pi_{1}^{\text {orb }}\left(D_{1}\right) \rightarrow 0
$$

As $D_{1}^{2}=\frac{1}{d}$, we have that

$$
\left.\pi_{1}\left(\partial U\left(D_{1}\right)\right)=\left\langle\alpha_{1}, \beta_{1}, x, \gamma_{1}\right|\left[\alpha_{1}, \beta_{1}\right] x=1, x^{d}=\gamma_{1}, \gamma_{1} \text { central }\right\rangle .
$$

As we mentioned before, $\alpha_{1}, \beta_{1}$ contract in $\mathbb{C} P^{2}-C$. So $x=1$ and $\gamma_{1}=x^{d}=$ 1. Therefore $\pi_{1}\left(X-\cup D_{i}\right)=1$. The orbifold fundamental group $\pi_{1}^{\text {orb }}(X)$ is the quotient with the conditions $\gamma_{i}^{m_{i}}=1$. In any case, $\pi_{1}^{\text {orb }}(X)=1$.

By [6, Corollary 10.2.11], a Smale-Barden manifold which admits a K-contact structure necessarily satisfies the $G-K$ condition, which means that, in terms of the expression (1):

- for every prime $p, t(p)=\#\left\{i \mid c\left(p^{i}\right)>0\right\} \leq k+1$,

- $i(M) \in\{0, \infty\}$; if $i(M)=\infty$ (M non-spin), then $t(2) \leq k$.

In [6, Question 10.2.1] it is asked whether a Smale-Barden manifold which satisfies the G-K conditon admits a Sasakian structure. Write

$$
\mathbf{t}(M)=\max \{t(p) \mid p \text { prime }\} \leq k+1 .
$$

The difficulty to obtain examples increases as we go to the upper bound, since we can always discard surfaces from the isotropy locus. The examples of $[8,23]$ are instances where the upper bound $\mathbf{t}(M)=k+1$ is achieved.

Note that the case $\mathbf{t}=0$ is that of torsion-free Smale-Barden manifolds, where we only have regular Sasakian structures, and all G-K manifolds admit Sasakian structures. The next case is $\mathbf{t}=1$, which is studied in detail in [25]. All G-K manifolds with $\mathbf{t}=1$ and $k \geq 1$ admit semi-regular Sasakian structures, and hence the manifolds admitting Sasakian and K-contact structures are the same. In the 
borderline case $\mathbf{t}=1, k=0$, the results in [25] are only partial and touch upon open questions on symplectic 4-manifold topology.

Write also

$$
\mathbf{c}(M)=\frac{1}{2} \max \left\{c\left(p^{i}\right)\right\}=\max \left\{g_{i}\right\} .
$$

in terms of the expression for $H_{2}(M, \mathbb{Z})$ given in Theorem 14. It is hard to get examples with low $\mathbf{c}(M)$ and $\mathbf{t}(M)=k+1, k=b_{2}(M)$. In [8] there is given an example with $\mathbf{c}(M)=3$, and in [22] there appears an example with $\mathbf{c}(M)=2$. Theorem 16 allows us to improve the bound.

Corollary 21. There is a simply connected 5-manifold $M$ admitting a (quasiregular) Sasakian structure with $\mathbf{t}(M)=b_{2}(M)+1$ and $\mathbf{c}(M)=1$.

Proof. Let $b \geq 1$ and consider the orbifold $X$ constructed in Theorem 16 . It contains $b$ disjoint complex curves of genus 1 spanning the homology. Choose a prime $p$ and consider coefficients $m_{i}=p^{i}$. Using Proposition 8 , we give $X$ an orbifold structure with isotropy locus given by the $D_{i}$ with coefficients $m_{i}$. We assign local invariants $b_{i}=1$, so that Theorem 12 allows to choose a Seifert bundle $M \rightarrow X$ whose Chern class is an orbifold Kähler form and $c_{1}(M / m)$ is primitive. Using Theorem 14 we have $H_{1}(M, \mathbb{Z})=0$. Note that $\pi_{1}^{\text {orb }}(X)=1$ by Proposition 20 , hence $\pi_{1}(X)=1$ and so $H_{1}(X, \mathbb{Z})=0$. By Theorem 14 we have

$$
H_{2}(M, \mathbb{Z})=\mathbb{Z}^{k} \oplus\left(\bigoplus_{i=1}^{k+1} \mathbb{Z}_{m_{i}}^{2}\right)
$$

with $m_{i}=p^{i}$ and $k=b-1$. To see that $M$ is Smale-Barden, we need to compute $\pi_{1}(M)$. There is an exact sequence $\mathbb{Z} \rightarrow \pi_{1}(M) \rightarrow \pi_{1}^{\text {orb }}(X)=1$, hence $\pi_{1}(M)$ is abelian. As $H_{1}(M, \mathbb{Z})=0$, it must be $\pi_{1}(M)=0$. This concludes the result.

Corollary 22. The manifold $M$ constructed in Corollary 21 is Sasakian quasiregular but does not admit a Sasakian semi-regular structure.

Proof. If $M$ admits a Sasakian semi-regular structure, then there is a Seifert fibration $M \rightarrow Y$, where $Y$ is a smooth Kähler surface. By Theorem $14, b_{1}(Y)=0$, $b_{2}(Y)=k+1=b$, and the ramification locus contains a collection of $b$ disjoint complex curves $D_{i}$ of genus $g_{i}=1$. By [8, Theorem 29], this is impossible (put differently, the Conjecture 3 holds in the case of curves of genus 1).

We end up this section by extending the result of Theorem 16 to higher genus.

Theorem 23. Take $d \geq 3$ and $g=\frac{(d-1)(d-2)}{2}$. Then, for any $b \geq 1$, there is $a$ Khler cyclic orbifold $X$ with $b_{1}=0, b_{2}=b$ and $b$ disjoint curves of genus $g$. In addition, $X$ can be constructed in such a way that $\pi_{1}^{\text {orb }}(X)=1$.

Proof. Consider inside the projective plane $\mathbb{C} P^{2}$, the family of curves, $C_{\lambda}, \lambda \in \mathbb{C}$, given by:

$$
C_{\lambda} \equiv\left\{\left[x_{0}, x_{1}, x_{2}\right] \in \mathbb{C} P^{2} \mid F_{\lambda}\left(x_{0}, x_{1}, x_{2}\right)=x_{0}^{d-1} x_{2}-x_{1}^{d}+\lambda x_{2}^{d}=0\right\} .
$$


These are all curves of degree $d$ and, therefore, they have genus $g=\frac{(d-1)(d-2)}{2}$. Now note that for $\lambda \neq 0, C_{\lambda}$ is smooth. Indeed, suppose $C_{\lambda}$ is not smooth at some point $\left[x_{0}, x_{1}, x_{2}\right]$. Then $0=\frac{\partial F_{\lambda}}{\partial x_{1}}=-d x_{1}^{d-1}$, from where $x_{1}=0$. Also $0=\frac{\partial F_{\lambda}}{\partial x_{0}}=$ $(d-1) x_{0}^{d-2} x_{2}$ from where either $x_{0}=0$ or $x_{2}=0$. Finally, $0=\frac{\partial F_{\lambda}}{\partial x_{2}}=x_{0}^{d-1}+d \lambda x_{2}^{d-1}$. Then in any case, $x_{0}=0, x_{2}=0$.

Now let us see that the curves $C_{\lambda}, \lambda \neq 0$, intersect at a single point with multiplicity $d^{2}$. Take $\lambda_{1} \neq \lambda_{2}$ and $p=\left[x_{0}, x_{1}, x_{2}\right] \in C_{\lambda_{1}} \cap C_{\lambda_{2}}$. Then

$$
\begin{aligned}
& x_{0}^{d-1} x_{2}-x_{1}^{d}+\lambda_{1} x_{2}^{d}=0, \\
& x_{0}^{d-1} x_{2}-x_{1}^{d}+\lambda_{2} x_{2}^{d}=0 .
\end{aligned}
$$

From here, $\lambda_{1} x_{2}^{d}=\lambda_{2} x_{2}^{d}$, which implies $x_{2}=0$ and subsequently $x_{1}=0$. Therefore $p=[1,0,0]$, and hence $C_{\lambda_{1}} \cdot C_{\lambda_{2}}=d^{2} p$.

Thus, selecting $b$ curves in the family $\left\{C_{\lambda}\right\}_{\lambda \neq 0}$, we get $b$ smooth curves $C_{1}, \ldots, C_{b}$ of genus $g$, each pair of them intersecting at the point $p_{0}=[1,0,0]$ with multiplicity $d^{2}$. Now the same argument as that in the proof of Theorem 16 produces the Khler cyclic orbifold $X$ with $b_{1}=0, b_{2}=b$ and $b$ disjoint curves $D_{1}, \ldots, D_{b}$ of genus $g$.

The proof of Proposition 20 works also in this case. We only need to choose the curves $C_{\lambda}$ close to each other (with numbers $\lambda$ very close together), so that the homotopy classes on each $C_{\lambda}$ can be pushed to $\partial U\left(C_{\lambda_{0}}\right)$, for a fixed $\lambda_{0}$. Take a basis $\alpha_{1}, \beta_{1} \ldots, \alpha_{g}, \beta_{g}$ of $\pi_{1}\left(C_{\lambda_{0}}\right)$. This basis can be given by loops well away from $p_{0}$. These can be contracted in $\mathbb{C} P^{2}-C_{\lambda_{0}}$, because they can be defined by vanishing cycles in a suitable Lefschetz fibration in which $C_{\lambda_{0}}$ is a fiber.

As in Corollary 21, the orbifold in Theorem 23 serves to construct a simply connected 5-manifold $M$ admitting a (quasi-regular) Sasakian structure with

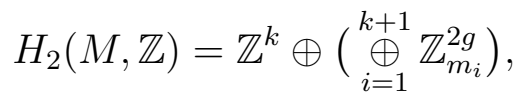

with $m_{i}=p^{i}$ and $k=b-1 \geq 0$, and $g=\frac{(d-1)(d-2)}{2}$ any triangular number. This manifold has $\mathbf{t}(M)=b_{2}(M)+1$ and $\mathbf{c}(M)=g$.

\section{Quasi-REgular vS SEMI-REgular}

Let us give an easy example of a quasi-regular Sasakian Smale-Barden manifold that cannot be semi-regular K-contact, which improves on Corollary 22.

Proposition 24. There is a cyclic Kähler orbifold $\bar{Y}$ with $b_{2}=1$ and an embedded curve $D$ of genus $g=2$.

Proof. Let $Y=H_{2}$ be the Hirzebruch surface with invariant $n=2$, that is $Y=$ $\mathbb{P}\left(\mathcal{O}_{\mathbb{C} P^{1}} \oplus \mathcal{O}_{\mathbb{C} P^{1}}(2)\right)$, which is a $\mathbb{C} P^{1}$-bundle over $\mathbb{C} P^{1}$. The zero section $\sigma$ has $\sigma^{2}=2$. Let $f$ be the fiber. Therefore the section at infinity $\sigma_{\infty} \equiv \sigma-2 f$ has 
$\sigma_{\infty}^{2}=-2$. The canonical class is $K_{Y}=-2 \sigma$ and the ample cone is generated by $\langle\sigma, f\rangle$.

We take a curve $D \equiv 2 \sigma+f$. This has genus 2 and $D^{2}=12$. It can be taken to be smooth. Let us give explicit equations. In the affine part $X^{o}=X-\sigma_{\infty}$, which is the total space of the line bundle $\mathcal{O}_{\mathbb{C P} P^{1}}(2)$ over $\mathbb{C} P^{1}$, we have a tautological coordinate $y$. Let $x$ be the affine coordinate of the basis. Then we take the equation

$$
y^{2} x+a(x) y+b(x)=0,
$$

where $a \in H^{0}\left(\mathbb{C} P^{1}, \mathcal{O}(3)\right), b \in H^{0}\left(\mathbb{C} P^{1}, \mathcal{O}(5)\right)$. It intersects the section $\sigma_{\infty}$ at one point $x=0, y=\infty$, and every fiber at two points. The condition to be smooth in the affine part is that the discriminant $\Delta(x)=a(x)^{2}-4 b(x) x$ has no double roots. At the point at infinity, we can take the coordinate $1 / y$, to see that the curve is smooth there.

Now we contract $\sigma_{\infty}$ and we get a cyclic orbifold $\bar{Y}$ with a point of order 2 . The projected curve $\bar{D}$ becomes a genus 2 curve going through the singular point.

Lemma 25. The orbifold fundamental group of the manifold in Proposition 24 is trivial if the isotropy coefficient of $\bar{D}$ is an integer $m$ with $\operatorname{gcd}(m, 6)=1$.

Proof. Consider the curves $D$ and $S=\sigma_{\infty}$ in $Y=H_{2}$. Let $\gamma, \delta$ be the loops around $D$ and $S$, and let $\alpha_{1}, \beta_{1}, \alpha_{2}, \beta_{2}$ the standard generators of $\pi_{1}(D)$. Then $\gamma$ and $\delta$ commute since $S, D$ intersect transversally at one point. Now in $\partial U(D)$, we have $\gamma^{12}=\left[\alpha_{1}, \beta_{1}\right]\left[\alpha_{2}, \beta_{2}\right]$, using that $D^{2}=12$, and $\gamma$ is central. Also $\delta^{2}=1$ in $\partial U(S)$. The loops $\alpha_{i}, \beta_{i}$ contract in $Y$, since it is simply-connected, hence it can be written in terms of $\gamma, \delta$ in $\pi_{1}(Y-(D \cup S))$. Therefore $\pi_{1}(Y-(D \cup S))=\pi_{1}(\bar{Y}-\bar{D})$ is generated by commuting loops $\gamma, \delta$ with $\gamma^{12}=1, \delta^{2}=1$.

The orbifold fundamental group $\pi_{1}^{\text {orb }}(\bar{Y})$ is a quotient of such group, by imposing the conditions $\delta^{2}=\gamma, \gamma^{m}=1$, where $m$ is the isotropy coefficient of $\bar{D}$. This implies the result.

Theorem 26. There is a quasi-regular Sasakian Smale-Barden manifold, which is not semi-regular K-contact.

Proof. Apply Theorem 12. Take an integer $m=m_{1} \operatorname{with} \operatorname{gcd}(m, 6)=1$ and local invariant $b_{1}=1$. Then use the Seifert bundle $M \rightarrow \bar{Y}$ with $c_{1}(M)=\frac{b_{1}}{m_{1}}\left[D_{1}\right]=$ $\frac{1}{m}\left[D_{1}\right]$. This is an orbifold Kähler form, hence $M$ is Sasakian. The class $c_{1}(M / m)=$ $\left[D_{1}\right]$ is primitive in $H^{2}(X-P, \mathbb{Z})$, since it pairs with the class $\sigma-2 f \in H_{2}(X-P, \mathbb{Z})$ giving 1 . Therefore we can apply Theorem 14 to prove that $H_{1}(M, \mathbb{Z})=0$ and

$$
H_{2}(M, \mathbb{Z})=\mathbb{Z}_{m}^{4}
$$

Using Lemma 25, we prove that $\pi_{1}(M)=1$ and hence $M$ is a Smale-Barden manifold. The manifold $M$ just constructed is quasi-regular Sasakian. It cannot be semi-regular K-contact, since in [25, Proposition 17] it is proved that this can only happen for $g=2$ being a triangular number. But this is not the case. 


\section{RATiONAL SYMPLECTIC 4-MANIFOLDS}

The purpose of this section is to show that (at least some of) the manifolds constructed in Section 3 cannot be semi-regular K-contact. This gives an example of quasi-regular Sasakian Smale-Barden $M$ that is not K-contact semi-regular in the case $b_{2}(M)>0$ (that is, not rational homology spheres). However, the proof is more technical than that of Theorem 26, since it uses Gromov-Witten and SeibergWitten theory for symplectic 4-manifolds.

Definition 27 ([20, Definition 2.2]). For a minimal symplectic 4-manifold $(X, \omega)$, let $K$ be the symplectic canonical class. We define the Kodaira dimension as:

$$
\kappa(X, \omega)= \begin{cases}-\infty & \text { if } K \cdot[\omega]<0 \text { or } K^{2}<0, \\ 0 & \text { if } K \cdot[\omega]=0 \text { and } K^{2}=0 \\ 1 & \text { if } K \cdot[\omega]>0 \text { and } K^{2}=0, \\ 2 & \text { if } K \cdot[\omega]>0 \text { and } K^{2}>0\end{cases}
$$

Corollary 28. If $(X, \omega)$ is a symplectic 4-manifold with $b_{1}=0$ and $K \cdot[\omega]<0$ then $X$ is rational (that is, symplectomorphic to a rational algebraic surface).

Proof. By [20, Theorem 2.4], if $(X, \omega)$ is minimal (that is, if it does not contain an embedded symplectic sphere $S$ with $\left.S^{2}=-1, K \cdot S=-1\right)$, then $\kappa(X, \omega)=-\infty$ if and only if it is rational or ruled. If $b_{1}=0$ and $X$ is ruled, then it is an $S^{2}$-bundle over $S^{2}$, that is a Hirzebruch surface, which is rational.

If $(X, \omega)$ is non-minimal, then there is a minimal symplectic manifold $\left(X^{\prime}, \omega^{\prime}\right)$ and a blow-up map $\pi: X \rightarrow X^{\prime}$. Suppose that $\pi$ is a single blow-up (the general case is done by repeating the argument). Note that $b_{1}\left(X^{\prime}\right)=0$. Let $E$ be the exceptional divisor, and $K, K^{\prime}$ the canonical classes. Then $K=K^{\prime}+E$ and $[\omega]=\left[\omega^{\prime}\right]-\alpha[E]$, for some $\alpha>0$. Then $K^{\prime} \cdot\left[\omega^{\prime}\right]=K \cdot[\omega]-\alpha<0$. Hence $X^{\prime}$ is rational, and so $X$ is also rational.

Lemma 29. Let $(X, \omega)$ be a compact symplectic 4-manifold, let $D$ be a symplectic surface with $[D]^{2}>0$. Then there is a symplectic form $\omega^{\prime}$ so that $\left[\omega^{\prime}\right]=[\omega]+\lambda[D]$, for any $\lambda>0$.

Proof. By the symplectic tubular neighbourhood theorem, we can assume that a neighbourhood of $D$ modelled in a complex manifold, that is $U \subset L$, where $\pi: L \rightarrow D$ is a holomorphic line bundle of degree $m=[D]^{2}>0$. We take the complex structure $J$ on $L$. The boundary of a unit circle bundle $S(L)$ in $L$ is a Sasakian manifold with a contact form $\eta$ such that $d \eta=\pi^{*}\left(\omega_{D}\right)$. Let $r$ be the radial coordinate of $L$ (we have fixed an hermitian metric). The form

$$
\beta=d\left((1 / 2) r^{2} \eta\right)=r d r \wedge \eta+(1 / 2) r^{2} \omega_{D}
$$

is a Kähler form for the cone, that is Kähler for $L$ except that it vanishes over the zero section. Note that $r^{2} \eta$ is a well-defined form on $L$, so $\beta$ is exact. 
Take a perturbation of $D$ as follows. We take a trivialization of $S(L)$ over all of $D$ but a point $p_{0}$, we lift to construct $D_{1}^{\prime}$ over $D-\left\{p_{0}\right\}$ at $r=\epsilon$, Then the boundary of $D_{1}^{\prime}$ is the fiber $S\left(L_{p_{0}}\right)$ with $m$ positive turns. We close it with $D_{2}^{\prime}$ which is $m$ copies of $B_{\epsilon}\left(L_{p_{0}}\right)$, and introduce the cycle $D^{\prime}=D_{1}^{\prime} \cup D_{2}^{\prime}$. Then

$$
0=\int_{D^{\prime}} \beta=\int_{D_{1}^{\prime}}(1 / 2) \epsilon^{2} \omega_{D}+\int_{B_{\epsilon}(0)} m r d r \wedge d \theta=(1 / 2) \epsilon^{2} \operatorname{area}(D)-\epsilon^{2} \pi m .
$$

In particular, we note that $d r \wedge d \theta<0$ in the fiberwise direction.

Now take a bump function $\rho(r)$ which is non-increasing, $\rho(r) \equiv 1$ for small $r$ and $\rho(r) \equiv 0$ for large $r$. Then take

$$
\Omega=d(\rho(r) \eta)=\rho^{\prime}(r) d r \wedge \eta+\rho \omega_{D}
$$

As $\rho^{\prime}(r) \leq 0$, this form is $J$-compatible semi-positive and compactly supported. The first term is positive since $d r \wedge \eta<0$. Note that this is not exact because $\rho(r) \eta$ does not extend over $D$. Now take $\omega+\lambda \Omega$, for $\lambda>0$, which solves the problem.

Theorem 30. If $(X, \omega)$ is a compact symplectic 4-manifold with $b_{1}(X)=0$, and $D$ is a symplectic surface with $[D]^{2}>0$ and $K \cdot D<0$, then $X$ is rational.

Proof. By Lemma 29, there is a symplectic form $\omega^{\prime}$ with $\left[\omega^{\prime}\right]=[\omega]+N[D]$, for $N>0$. Now

$$
K \cdot\left[\omega^{\prime}\right]=K \cdot[\omega]+N K \cdot[D]<0
$$

for $N \gg 0$ large enough. Now by Corollary $28,\left(X, \omega^{\prime}\right)$ must be rational.

Corollary 31. Suppose that $X$ is a symplectic 4-manifold with $b_{1}(X)=0$, and with disjoint surfaces $D_{i}$ of genus 1 and spanning the homology $H_{2}(X, \mathbb{Q})$. Then $X$ is rational.

Proof. As $X$ is symplectic then $b^{+} \geq 1$. So there must be one of the surfaces, say $D_{1}$, with $D_{1}^{2}>0$. As $D_{1}$ is a torus, then $K \cdot D_{1}=-D_{1}^{2}<0$. Apply now Theorem 30 .

Proposition 32. Suppose that $X$ is a symplectic 4-manifold with $b_{1}=0$, and with disjoint surfaces $D_{i}$ of genus 1 and spanning the homology $H_{2}(X, \mathbb{Q})$. Then $b_{2} \neq 2$.

Proof. Suppose that $b_{2}=2$. By Corollary 31, $X$ is rational, hence it must be a Hirzebruch surface $H_{n}, n \geq 0$. Let $D_{1}, D_{2}$ be the disjoint symplectic genus 1 curves. Then

$$
D_{1} \cdot D_{2}=0, \quad D_{1} \cdot\left(K+D_{1}\right)=0
$$

imply by inspection of the intersection matrix that

$$
D_{1}+D_{2}+K=0 .
$$

Let $\sigma, f$ be the basis of $H_{2}(X, \mathbb{Z})$ given by the special section $\sigma$ and the fiber $f$ of the fibration, with

$$
\sigma^{2}=-n, \sigma \cdot f=1, f^{2}=0
$$


Then $K=-2 \sigma-(n+2) f$. Write $D_{1}=a \sigma+b f$, with $a, b \in \mathbb{Z}$. Interchanging $D_{1}$ and $D_{2}$ if necessary, we can assume $a \leq 1$ by (4). The equation $D_{1} \cdot D_{2}=0$ simplifies to

$$
(a-1)(a n-2 b)=-2 a .
$$

Thus $a \neq 0,1$, so $a \leq-1$. Since (5) is an integer relation, we use $\operatorname{gcd}(a, a-1)=1$ to infer that $(a-1) \mid 2$, hence $a=-1$. Then (5) gives $n+2 b=1$, and hence $b \leq 0$. Consider the Khler class $[\omega]=x \sigma+y f$ with $x>0, y>n x$ (since the surfaces $\sigma$ and $f$ are symplectic, hence they pair positively with $[\omega])$. But then we find

$$
0<[\omega] \cdot D_{1}=\underbrace{(n x-y)}_{<0}+b x .
$$

Since $b<0$ we obtain the required contradiction.

Remark 33. Note that symplectic surfaces in a symplectic 4-manifold can pair negatively. Therefore, we cannot assume that $D_{1} \cdot f \geq 0$ in the proof above, which would simplify the argument.

Also note that in the complex case, equation (4) leads to a contradiction since it would provide an exact sequence $0 \rightarrow \mathcal{O}(K) \rightarrow \mathcal{O} \rightarrow \mathcal{O}_{D_{1}} \oplus \mathcal{O}_{D_{2}} \rightarrow 0$. Using that $H^{0}(K)=0, H^{1}(K)=0$, we would get that $H^{0}(\mathcal{O})=H^{0}\left(\mathcal{O}_{D_{1}}\right) \oplus H^{0}\left(\mathcal{O}_{D_{2}}\right)=\mathbb{C}^{2}$, which is not true. However, for almost complex 4-manifolds there is no analogue of this cohomology theory.

Now we complete the proof that the manifold $M$ of Corollary 22 is not semirregular K-contact for $k=1$.

Theorem 34. If a Smale-Barden manifold $M$ has $H_{2}(M, \mathbb{Z})=\mathbb{Z} \oplus\left(\oplus_{i=1}^{2} \mathbb{Z}_{p^{i}}^{2}\right)$, then it cannot be K-contact semi-regular.

Proof. Suppose that $M$ is K-contact semi-regular. Then there is a Seifert fibration $\pi: M \rightarrow Y$, where $Y$ is a smooth symplectic 4-manifold with $b_{1}(Y)=0, b_{2}(Y)=$ $b=k+1=2$, with a collection of disjoint smooth symplectic embedded surfaces $D_{i}$ of genus 1 , and spanning the homology $H_{2}(Y, \mathbb{Q})$. This is impossible by Proposition 32 .

Remark 35. Note that we have no chance to extend Theorem 34 to the SmaleBarden manifold of (3) with $k=1$ and $g>1$. Our proof relies heavily on the rationality of symplectic 4-manifolds, proved in Corollary 31, which hinges on the fact that the surfaces are of genus 1 .

\section{Null Sasakian Structures}

6.1. A description of null Sasakian structures. A smooth K3 surface is a simply connected complex surface with trivial canonical class $K_{X}$. The condition $K_{X}=0$ is equivalent to the existence of a nowhere vanishing holomorphic 2 -form 
$\omega_{X}$. To define the orbifold version of this definition, we need orbifold homology $H_{i}^{\text {orb }}(X)$ and the orbifold canonical class $K_{X}^{\text {orb }}$ (see [6, Chapter 4$]$ ).

Definition 36. An orbifold K3-surface is a cyclic orbifold $X$ such that $H_{1}^{\text {orb }}(X)=0$ and $K_{X}^{\text {orb }}=0$.

Proposition 37 ([18, Proposition 10.2]). Let $\pi: M \rightarrow\left(X, \Delta=\sum_{i}\left(1-\frac{1}{m_{i}}\right) D_{i}\right)$ be a Seifert bundle with $M$ smooth. Assume that $H_{1}(M, \mathbb{Z})=0$ and that the orbifold $(X, \Delta)$ is a Calabi-Yau orbifold, that is $K_{X}+\Delta$ is numerically trivial. Then $K_{X}$ is trivial (and $\Delta=0$ ).

In addition, $M$ is simply connected if and only if $\pi_{1}^{\text {orb }}(X)=1$ by [18, Thm. 9.1].

Proposition 38 ([18, Corollary 10.4]). Let $\pi: M \rightarrow(X, \Delta)$ be a 5-dimensional Seifert bundle, $M$ smooth. Assume that $H_{1}(M, \mathbb{Z})=0$, and that $(X, \Delta)$ is a Calabi-Yau orbifold. Then

(1) the minimal resolution of $X$ is a K3-surface,

(2) $M$ is homeomorphic to the connected sum of at most 21 copies of $S^{2} \times S^{3}$.

Theorem 39 ([6, Theorem 10.3.8]). If a Smale-Barden manifold $M$ admits a null Sasakian structure, then

(1) any null Sasakian structure is quasi-regular, and, therefore, $M$ admits a structure of a Seifert bundle over an orbifold K3 surface,

(2) $2 \leq b_{2}(M) \leq 21$,

(3) if $b_{2}(M)=21$, the null structure is regular, that is, the Seifert bundle $M \rightarrow X$ is a smooth principal circle bundle over a K3 surface,

(4) $M$ is spin,

(5) $\pi_{1}^{\mathrm{orb}}(X)=1$.

Proof. It follows from Proposition 38 and the discussion around it in a straightforward manner (see [6, Section 10.3.2]).

Theorem 40 ([6, Corollary 10.3.11] and [12, Theorem A]). Any $M=\#_{k}\left(S^{2} \times S^{3}\right)$ with $2 \leq b_{2}(M) \leq 21$ admits a null Sasakian structure, except, possibly, $b_{2}(M)=2$.

Proof. The proof for all cases except $k=17$ is based on the list of examples of orbifold K3 surfaces in [28]. These are hypersurfaces in weighted projective spaces. In the case $k=17$ the author of [12] goes along the similar lines using the list of weighted complete intersections in [16]. The second Betti numbers are calculated in [6, Example 10.3.10] and in [12], and it appears that all $k$ can be realized except $k=2$. 
6.2. Preparatory work to prove Theorem 5. In order to construct a null Sasakian structure on a Smale-Barden manifold $M$ with $b_{2}(M)=2$, we need a cyclic K3 orbifold $X$ with $b_{2}(X)=3$ and with the property $\pi_{1}^{\text {orb }}(X)=1$. This follows from Theorem 14. Thus, we begin with a construction of such an orbifold. We will use the method of lattices going back to Pyatetskii-Shapiro and Shafarevich [27] and developed in [26] combined with methods of calculation of the fundamental groups of smooth parts of K3 surfaces with cyclic singularities (that is, $\pi_{1}^{\text {orb }}(X)$ ) from $[17,33]$. We understand a lattice as a free $\mathbb{Z}$-module endowed with a nondegenerate symmetric bilinear form with values in $\mathbb{Z}$. If $R$ is a lattice and $x, y \in R$, we write $x \cdot y$ for the value of this form on $(x, y)$. Assume that $\alpha \in R$ satisfies $\alpha^{2}=-2$. Then $\alpha$ determines an automorphism $s_{\alpha}: R \rightarrow R$ by the formula

$$
s_{\alpha}(x)=x+(x \cdot \alpha) \alpha .
$$

Assume that the bilinear form $(x, y) \rightarrow x \cdot y$ on $R$ is negative definite, and that $R$ is generated by elements of square $(-2)$. Then the group generated by all $s_{\alpha}$ is the Weyl group, and free generators of square $(-2)$ constitute a root system, and one can associate with $R$ a Dynkin diagram in a standard way (see [26] for a more detailed account). In particular, it is known that such lattices correspond to the Dynkin diagrams which are disjoint unions of connected Dynkin diagrams of types $A_{k}, k \geq 1, D_{l}, l \geq 4, E_{6}, E_{7}, E_{8}$. Thus, we will call $R$ a root lattice.

Let $X$ be a smooth $\mathrm{K} 3$ surface. Then $H^{2}(X, \mathbb{Z})$ is an even unimodular lattice with the intersection form of signature $(3,19)$ as the bilinear form in the definition. Consider complex elliptic surfaces $f: X \rightarrow \mathbb{C} P^{1}$ with a section $O$. The NéronSeveri lattice $N S(X)$ of $X$ is defined as $H^{1,1}(X) \cap H^{2}(X, \mathbb{Z})$. For elliptic surfaces over $\mathbb{C} P^{1}, N S(X)$ coincides with the Picard lattice $\operatorname{Pic}(X)$. The cohomology classes of $O$ and a generic fiber of $f$ generate a sublattice $U_{f}$ of rank 2 . Since $U_{f}$ turns to be a hyperbolic lattice, there is a decomposition $N S(X)=U_{f} \oplus W_{f}$ for some negative-definite even lattice $W_{f}$. The Mordell-Weil group $M W_{f}$ of $X$ is understood as $N S(X) / T$, where $T$ is generated by $O$ and the fiber components [32].

In the sequel, we require that $X$ is extremal, that is, the Picard number $\rho(X)=$ 20 and the Mordell-Weil group $M W_{f}$ is finite. Such surfaces are classified in [33]. We will need a more detailed account of this classification. Let $R_{f}$ be the (finite) set of points $v \in \mathbb{C} P^{1}$ such that $f^{-1}(v)$ is reducible. For a point $v \in R_{f}$ the notation $f^{-1}(v)^{\#}$ means the union of reducible components of $f^{-1}(v)$ that are disjoint from $O$. It has been known since Kodaira's work that the cohomology classes of irreducible components of $f^{-1}(v)^{\#}$ form a negative definite root lattice $S_{f, v}$. The sum of disjoint components of the Dynkin diagrams corresponding to $S_{f, v}$ will be called the type $\tau\left(S_{f, v}\right)$ of the lattice. Define the formal sum of the types of such lattices:

$$
\Sigma_{f}=\sum_{v \in R_{f}} \tau\left(S_{f, v}\right)
$$


and denote by $S_{f}$ the direct sum of $S_{f, v}$.

Define

$$
\mathrm{eu}\left(\Sigma_{f}\right)=\sum_{l \geq 1} a_{l}(l+1)+\sum_{m \geq 4} d_{m}(m+2)+\sum_{n=6}^{8} e_{n}(n+2)
$$

where $a_{l}, b_{m}$ and $e_{n}$ denote the numbers of the connected components of the Dynkin diagrams of each type. Denote by $\Gamma_{f}$ the union of the zero section and all irreducible components of $f^{-1}(v), v \in R_{f}$. For a point $v \in R_{f}$, we denote the total fiber of $f$ over $v$ by

$$
\sum_{i=1}^{r_{v}} m_{v, i} C_{v, i}
$$

where $m_{v, i}$ is the multiplicity of the irreducible component $C_{v, i}$ of $f^{-1}(v)$. It is known that $M W_{f}=W_{f} / S_{f}$ (cf. [32, Prop. 6.42] or [33, Lem. 2.5]).

Theorem 41 ([33, Claim 2, p. 36]). Assume $M W_{f}=0$. Suppose that a configuration $\Gamma \subset \Gamma_{f}$ satisfies the following conditions:

(Z1) the number of $v \in R_{f}$ such that $C_{v, i} \subset \Gamma$ holds for any $C_{v, i}$ with $m_{v, i}=1$ is at most one,

(Z2) $\mathrm{eu}\left(\Sigma_{f}\right) \leq 23$.

Then $\pi_{1}(X-\Gamma)=1$.

6.3. Proof of Theorem 5. We begin with a construction of a K3 orbifold with $b_{2}(X)=3$ and $\pi_{1}^{\text {orb }}(X)=1$. In [35], there is a Weierstrass form given for an elliptic K3 surface $X^{\prime}$, with a fibre of Kodaira type $\mathrm{I}_{19}$, i.e. a cycle of $19(-2)$ curves. Exactly one fibre component, say $\Theta_{0}$ connects with the zero section of the fibration, which provides another $(-2)$-curve $O$. Omitting one of the two fibre components adjacent to $\Theta_{0}$, we derive an $A_{19}$ configuration $\Gamma$ of $(-2)$-curves given by $O, \Theta_{0}, \Theta_{1}, \ldots, \Theta_{17}$. This configuration is a part of the basis of $\operatorname{Pic}\left(X^{\prime}\right)$, since one can just add the remaining fiber component. Since $\mathrm{eu}\left(A_{19}\right)=20$ and $M W_{f}=W_{f} / S_{f}=0$, we see that $X^{\prime}$ satisfies the conditions $(Z 1)$ and $(Z 2)$ of Theorem 41. Thus, $\pi_{1}\left(X^{\prime}-\Gamma\right)=1$.

Contracting the 19 curves $O, \Theta_{0}, \Theta_{1}, \ldots, \Theta_{17}$ successively, we obtain an orbifold K3 surface $X$ with singularity $P$ of type $A_{19}, b_{2}(X)=3$ and

$$
\pi_{1}\left(X^{\prime}-\Gamma\right)=\pi_{1}(X-P)=\pi_{1}^{\text {orb }}(X)=1 .
$$

Now we take a Seifert bundle $M \rightarrow X$ with primitive Chern class $c_{1}(M) \in$ $H^{2}(X-P, \mathbb{Z})$. This can be arranged by picking first a primitive class in $H^{2}(X-$ $P, \mathbb{Z}$ ), then constructing the line bundle $M \rightarrow X-P$, and finally extending it as a Seifert bundle $\pi: M \rightarrow X$ across $P$. Moreover, we can assure that $c_{1}(M)$ is a Khler class, so that Theorem 13 applies to produce a Sasakian structure on $M$. As clearly $H_{1}(X, \mathbb{Z})=0$, we have constructed a Seifert bundle satisfying the assumptions of 
Theorem 14. From these we get $H_{1}(M, \mathbb{Z})=0$, and Theorem 5 follows using the fact that $\pi_{1}^{\text {orb }}(X)=1$ implies $M$ to be simply connected, so $M=\#_{2}\left(S^{2} \times S^{3}\right)$.

Remark 42. In [33] the authors use the notation $\Delta$ instead of our $\Gamma$ (in this article $\Delta$ is a branch divisor).

Remark 43. One can also work with other K3 surfaces $X^{\prime}$, such as the one from [33, Remark 4.10].

6.4. Remarks on the classification of null Sasakian structures. The classification of null Sasakian structures amounts to a classification of orbifold K3 surfaces with cyclic singularities satisfying the conditions $\pi_{1}^{\text {orb }}(X)=1$. In general this may be out of reach, however, in case $\#_{2}\left(S^{2} \times S^{3}\right)$, one needs to classify cyclic orbifolds $X$ with $b_{2}(X)=3$ and trivial orbifold fundamental group. For example, it is concievable that one can classify extremal elliptic K3 fibrations with configurations of $(-2)$ curves of type $A$, using $[17,33,31]$. For K3 surfaces of degree 2 and 6 , this has been achieved in [37], resp. [36] (without considering fundamental groups).

\section{REFERENCES}

[1] D. Barden, Simply-connected five-manifolds, Ann. Math. 82(1965), 365-385.

[2] W. Barth, K. Hulek, C. Peters, A. Van de Ven, Compact Complex Surfaces, Springer, 2004.

[3] G. Bazzoni, I. Biswas, M. Fernández, V. Muñoz, A. Tralle, Homotopic properties of Kähler orbifolds, In: Special Metrics and Group Actions in Geometry, Springer INdAM Series 23, Springer, 23-57.

[4] I. Biswas, M. Fernández, V. Muñoz, A. Tralle, On formality of orbifolds and Sasakian manifolds, J. Topology 9(2016), 161-180.

[5] F. Atiyah, R. Bott. A Lefschetz fixed point formula for elliptic complexes. I, Ann. of Math. (2) 86(1967), 374-407.

[6] C. Boyer, K. Galicki, Sasakian Geometry, Oxford, 2007.

[7] C. Boyer, K. Galicki, P. Matzeu, On eta-Einstein Sasakian geometry, Commun. Math. Phys. 262(2006), 177-208.

[8] A. Cañaz, V. Muñoz, J. Rojo, A. Viruel, A K-contact simply connected 5-manifold without Sasakian structures, arxiv: 1911.08901

[9] B. Cappelletti-Montano, A. De Nicola, I. Yudin, Hard Lefschetz theorem for Sasakian manifolds, J. Diff. Geom. 101(2015), 47-66.

[10] B. Cappelletti-Montano, A. De Nicola, J. C. Marrero, I. Yudin, Examples of compact K-contact manifolds with no Sasakian metric, Int. J. Geom. Methods Modern Phys. 11(2014), 1460028.

[11] B. Cappelletti-Montano, A. De Nicola, J. C. Marrero, I. Yudin, A non-Sasakian Lefschetz K-contact manifold of Tievsky type, Proc. Amer. Math. Soc. 144(2016), 54575468.

[12] J. Cuadros Valle, Null Sasaki $\eta$-Einstein structures in 5-manifolds, Geom. Dedicata 169(2014), 343-359.

[13] R. Gompf, A. Stipsicz, 4-Manifolds and Kirby Calculus, AMS, Providence, RI, 2004.

[14] B. Hajduk, A. Tralle, On simply connected compact K-contact non-Sasakian manifolds, J. Fixed Point Theory Appl. 16(2014), 229-241. 
[15] K. Hulek, M. Schütt, Enriques surfaces and Jacobian elliptic surfaces, Math. Z. 268(2011), 1025-1056.

[16] A. Iano-Fletcher, Working with weighted complete intersections, explicit birational geometry of 3-folds, in: London Math. Soc. Lecture Notes Ser., vol. 281, Cambridge Univ. Press, Cambridge, 2000, 101-173.

[17] J. Keum, D-Q. Zhang, Fundamental groups of open K3 surfaces, Enriques surfaces and Fano 3-folds, J. Pure Appl. Algebra 170(2002), 67-91

[18] J. Kollár, Einstein metrics on 5-dimensional Siefert bundles, J. Geom. Anal. 15(2005), 445-476.

[19] J. Kollár, Circle actions on simply connected 5-manifolds, Topology 45(2006), 643-672.

[20] T-J. Li, Symplectic 4-manifods with Kodaira dimension zero, J. Diff. Geom. 74(2006), 321-352.

[21] R. Miranda, U. Persson, On extremal rational elliptic surfaces, Math. Z. 193(1986), 537-558.

[22] V. Muñoz, Gompf connected sum for orbifolds and K-contact Smale-Barden manifolds, arXiv:2003.07319

[23] V. Muñoz, J. A. Rojo, A. Tralle, Homlogy Smale-Barden manifolds with K-contact and Sasakian structures, Internat. Math. Res. Notices, doi.org/10.1093/imrn/rny205

[24] V. Muñoz, A. Tralle, Simply connected K-contact and Sasakian manifolds of dimension 7, Math. Z. 281(2015), 457-470.

[25] V. Muñoz, A. Tralle, On the classification of Smale-Barden manifolds with Sasakian structures, arxiv:2002.00457

[26] V. Nikulin, Quotient groups of groups of automorphisms of hyperbolic forms by subgroups generated by reflections. Algebro-geometric applications, Itogi Nauki i Techniki, Ser. Sovrem. Probl. Mat., 1981, vol. 18, 3-114.

[27] I. Pyatetskii-Shapiro, I. Shafarevich, A Torelli theorem for algebraic surfaces of type K3, Math. USSR - Izv. 5(1972), 547-588.

[28] M. Reid, Canonical 3-folds, Journées de Geometrie Algebrique d'Angers, 1980, 273310.

[29] M. Reid, The Du Val singularities, https ://homepages . warwick.ac.uk/m̃asda/surf/more/DuVal.pdf

[30] R. Rukimbira, Chern-Hamilton conjecture and K-contactness, Houston J. Math. 21(1995), 709-718.

[31] M. Schütt, Divisibilities among nodal curves, Math. Res. Letters 25(2018), 1359-1368.

[32] M. Schütt, T. Shioda, Mordell-Weil lattices, Springer, Berlin, 2019.

[33] I. Shimada, D-Q. Zhang, Classification of extremal elliptic K3 surfaces and fundamental groups of open K3 surfaces, Nagoya Math. J. 161(2001), 23-54.

[34] S. Smale, On the structure of 5-manifolds, Ann. Math. 75(1962), 38-46.

[35] T. Shioda, The elliptic K3 surfaces with a maximal singular fibre, C. R. Acad. Sci. Paris, Ser. I, 337(2003), 461-466.

[36] A-K. Stegmann, Cubic fourfolds with ADE-singularities and K3 surfaces, $\mathrm{PhD}$ thesis (2020), Leibniz Universität Hannover.

[37] J-G. Yang, Sextic curves with simple singularities, Tohoku Math. J. (2) 48(1996), 203-227. 
Departamento de Álgebra, Geometría y Topología, Facultad de Ciencias, Universidad de Málaga, Campus de Teatinos s/n, 29071 Mlaga, Spain

E-mail address: alejandro.cm.95@uma.es

Departamento de Álgebra, Geometría y Topología, Facultad de Ciencias, Universidad de Málaga, Campus de Teatinos s/n, 29071 Mlaga, Spain

E-mail address: vicente.munoz@uma.es

Institut für Algebraische Geometrie, Gottfried Wilhelm Leibniz Universität Hannover, Welfengarten 1, 30167 Hannover, Germany

E-mail address: schuett@math.uni-hannover.de

Faculty of Mathematics and Computer Science, University of Warmia and Mazury, SŁoneczna 54, 10-710 Olsztyn, Poland

E-mail address: tralle@matman.uwm.edu.pl 